\title{
UV habitable zones around M stars
}

\author{
Andrea P. Buccino ${ }^{a}$ Guillermo A. Lemarchand ${ }^{\mathrm{b}}$ \\ Pablo J. D. Mauas \\ ${ }^{a}$ Instituto de Astronomía y Física del Espacio (CONICET), C.C. 67 Sucursal 28, \\ C1428EHA-Buenos Aires Argentina \\ ${ }^{\mathrm{b}}$ Facultad de Ciencias Exactas y Naturales, Universidad de Buenos Aires, C.C. 8 - \\ Sucursal 25, C1425FFJ Buenos Aires Argentina and Instituto Argentino de \\ Radioastronomía (CONICET), C.C. 5, 1894, Villa Elisa, Buenos Aires, Argentina
}

\begin{abstract}
During the last decade there was a change in paradigm, which led to consider that terrestrial-type planets within liquid-water habitable zones (LW-HZ) around M stars can also be suitable places for the emergence and evolution of life. Since many dMe stars emit large amount of UV radiation during flares, in this work we analyze the UV constrains for living systems on Earth-like planets around dM stars. We apply our model of UV habitable zone (UV-HZ, Buccino et al. 2006) to the three planetary systems around dM stars (HIP 74995, HIP 109388 and HIP 113020) observed by IUE and to two M-flare stars (AD Leo and EV Lac). In particular, HIP 74995 hosts a terrestrial planet in the LW-HZ, which is the exoplanet that most resembles our own Earth. We show, in general, that during the quiescent state there would not be enough UV radiation within the LW-HZ to trigger the biogenic processes and that this energy could be provided by flares of moderate intensity, while strong flares do not necessarily rule-out the possibility of life-bearing planets.
\end{abstract}

Key words: Extrasolar planets, Prebiotic environments, Exobiology, Ultraviolet Observations.

\section{Introduction}

Dwarfs of spectral type M (dM stars) constitute $75 \%$ of main sequence stars and, even having relative low masses, they contribute more than any other spectral type

Email addresses: abuccino@iafe.uba.ar (Andrea P. Buccino), lemar@correo.uba.ar (Guillermo A. Lemarchand), pablo@iafe.uba.ar (Pablo J. D. Mauas). 
to the total stellar mass of the galaxy (Rodonó, 1986). M stars are much smaller in mass than the Sun (between 0.08 and $0.5 M_{\odot}$ ) and their hydrogen burning lifetimes are much longer. Their lifetimes range from 50 Gyr to several trillion years (Laughlin et al., 1997) with a very slow change in their emitted flux. They also have relatively low stellar temperatures $\left(2400 K \lesssim T_{\text {eff }} \lesssim 3900 K\right)$. In the visual range, a typical M0 V spectrum represents only $1.9 \%$ of the solar flux, while at the infrared band, the relation is $16 \%$. On the other extreme (e.g. M9 V) the values are respectively $1.4 \times 10^{-14} \%$ and $0.13 \%$. Therefore, the balance of radiation of M stars is very different from our Sun.

Using numerical simulations, Wetherill (1996) showed that planets are likely to form in the habitable zones (HZ) of M dwarfs. In the last years, several new proposals to enhance the detection of planets around M dwarfs were promoted (Endl et al. 2003, Deeg 2004). However, recent surveys have only detected nine planetary systems around M dwarfs (Schneider, 2007). In particular, Udry et al. (2007) detected two super-Earth planets around the HIP 74995 system. One of them (Gl581c) is of great interest since it has a mass of $5.1 \mathrm{M}_{\oplus}$ (the lowest mass found for an exoplanet to date) and resides in the liquid-water habitable zone (LW-HZ). Udry et al. (2007) affirm that Gl581c is the exoplanet yet discovered that most resembles the Earth. However, von Bloh et al. (2007) calculated the habitable zone constrained by the levels of biological productivity on Gl581c's surface and found this planet too close to the parent star to host life.

Habitable planets around the low-mass $M$ stars would have some significant differences to Earth. In particular, most planets within the liquid water habitable zones defined in Kasting et al. (1993) are probably tidally locked, with the same side always facing the central star (Dole 1964, Kasting et al. 1993, Joshi 2003). Early arguments assumed that atmospheric volatiles would freeze in the dark side and boil off on the side of the planet facing the star. In this way, the the atmospheric pressure at the surfacewould be orders of magnitude below present-dayEarth and far below the minimum pressure at which liquid water can exist. However, according to different models of increasing complexity developed by Haberle et al. (1996), Joshi et al. (1997), Heath et al. (1999) and Joshi (2003), atmospheric heat transport could prevent freezing on the dark side of the planet. Therefore, it could be possibleto have habitable synchronously rotating planets where liquid water can exist.

Another important characteristic is that starspots may induce rotationally-modulated variations of several percent in the M-stellar radiation on time-scales of days (Rodonó 1986, Benedict et al. 1998, Kiraga and Stepien 2007). Although large starspots may cause a significant decrease in the stellar brightness, an atmosphere with a pressure of 1 bar at the surface of the planet would not freeze (Joshi et al., 1997). Atmospheric collapse would occur only if the planet were at the coldest $\mathrm{CO}_{2}$ condensation end of the habitable zone (Kasting et al., 1993).

In a recent review, Tarter et al. (2007) concluded that M dwarf stars may indeed be viable hosts for planets on which the origin and evolution of life can occur. They have also found a number of planetary processes such as cessation of geothermal activity, 
or thermal and non-thermal atmospheric loss processes that may limit the duration of planetary habitability to times far shorter than the lifetime of the $\mathrm{M}$ dwarf star.

Scalo et al. (2007) describe several sources of environmental fluctuations generated by dMe flares with strong influence on their habitability conditions. These include short-term variations in UV and blue radiation at the surface, effects due to alterations of the atmospheric photochemistry, heating of the atmosphere and surface by the effects of strong flares and energetic particles that could have intermittent effects on ozone and other chemistry.

It is well known that in the Sun there is a close correlation between strong flares and coronal mass ejections (CME). Khodachenko et al. (2007) analyzed the impact of stellar CMEs on Eath-like planets around active M stars. They found that those exoplanets within the habitable zones close $(<0.01 \mathrm{AU})$ to the dMe star would be exposed to the effects of CMEs for a long period. Since most of these exoplanets are tidally locked, their intrinsic magnetic moments are weak and the magnetosphere can be compressed under the action of CME plasma flow. This interaction would cause strong atmospheric erosion on Earth-like exoplanets.

In particular, UV radiation between 200 and $300 \mathrm{~nm}$ is known to inhibit photosynthesis, induce DNA destruction and cause damage to a wide variety of proteins and lipids (Lindberg and Horneck 1991, Cockell 1998). On the other hand, UV radiation is thought to have played an important role in the origin of life (Toupance et al. 1977, Ehrenfreund et al. 2002). It is usually considered one of the most important energy sources on the primitive Earth for the synthesis of many biochemical compounds. Based on these considerations and the Principle of Mediocrity 1 , in Buccino et al. (2006) we defined the boundaries of an ultraviolet habitable zone (UV-HZ). In that work, we also analyzed the UV-HZ on solar-type stars with exoplanets observed by the International Ultraviolet Explorer (IUE) satellite.

In the present work, we extend our analysis by considering the UV constrains for the emergence and evolution of living systems for all the $\mathrm{M}$ stars with exoplanets that have been observed by IUE.We compare these results with the LW-HZ. Since many $\mathrm{dM}$ flare stars emit large amounts of ultraviolet radiation and X-rays during flares, we also analyze how the UV Habitable Zones behave with the presence of moderate and strong flares.

In $\$ 2$ we describe the methodologies used to perform the observations and data analysis. In 93 we apply our model to the dM planetary systems and to two dM flare stars and in $\$ 4$ we present a discussion of the results.

\footnotetext{
1 The so-called Principle of Mediocrity proposes that our planetary system, life on Earth and our technological civilization are about average and that life and intelligence will develop by the same rules of natural selection wherever the proper conditions and the needed time are given (von Hoerner, 1961).
} 


\section{Observations}

To date (June 2007) nine dM stars were found hosting planetary systems. Three of them were observed by IUE: HIP 74995 (M3V), HIP 109388 (M3.5V) and HIP 113020 (M4V). In Table 1 we list some physical parameters of the host stars and their corresponding planets. The flare stars AD Leo (M3.5Ve) and EV Lac (M4.5Ve) were also studied to analyze the influence of flares on living systems.

\section{[Table 1]}

To study the influence of near UV radiation to exoplanets around M stars, we use IUE low-(0.6 $\mathrm{nm}$ resolution) and high-dispersion $(\lambda / \Delta \lambda \sim 10000)$ spectra, taken by the long wavelength cameras (LWP and LWR) in the range 185-340 nm. The spectra are available inthe IUE public library (at http://ines.laeff.esa.es/cgi-ines/IUEdbsMY), and have been calibrated using the NEWSIPS (New Spectral Image Processing System) algorithm. The internal accuracy of the high-resolution calibration is around $4 \%$ (Cassatella et al. 2000) and the errors of the low- dispersion spectra in the absolute calibration are around 10-15\% (Massa and Fitzpatrick, 1998).

For each of the three dM stars of our sample, only one IUE low-resolution spectra is available. On the other hand, for EV Lac there are 54 IUE low-resolution spectra in the long-wavelength range, and for AD Leo 64 low- and high-resolution spectra in the same wavelength range.

\section{[Figure 1]}

To illustrate the dependence of UV stellar radiation on its spectral class, in Fig. 1 we show a selection of IUE spectra scaled at 1 AU corresponding to the following stars of different spectral types: HD 9826 (F8V), HD 3651 (K0V) and the solar twin 18 Scorpii $(\mathrm{G} 2 \mathrm{~V})$, together with the stars under study here. We also include two IUE spectra of the very active star $\mathrm{AD}$ Leo $(\mathrm{M} 3.5 \mathrm{Ve})$ in the quiescent state and at the maximum of a strong flare. It is worth mentioning that HIP 113020 (M4V) was reported as an inactive M star by Johns-Krull and Valenti (1996), HIP 74995 (M3V) presents signatures of weak chromospheric activity (Bonfils et al., 2005) and HIP 109388 (M3.5V) was reported as a middle-aged dwarf star of low activity (Butler et al., 2006). Therefore, the dM spectra in Fig 1 show different levels of stellar activity.

We can see in Fig. 1 that the level of UV radiation is much lower in dM stars than in $\mathrm{G}$ stars. In particular, due to their low effective temperature, dM spectra are dominated by molecular absorption bands. 


\section{$3 \quad$ Habitable zones around M stars}

We computed the LW-HZ (Kasting et al. 1993) and the UV-HZ as described in Buccino et al. (2006) for all the M star with exoplanets observed by IUE. We refer the reader to the latter paper for details. In what follows we summarize the expressions we used in Buccino et al. (2006).

As mentioned in \$1, it is believed that UV radiation played an important role in the Earth biogenic processes (Toupance et al. 1977, Ehrenfreund et al. 2002). Consequently, there should be a minimum number of UV photons for the chemical synthesis of complex molecules to happen also in exoplanets. Based on the Principle of Mediocrity, we set the outer limit of the UV-HZ $\left(d_{\text {out }}\right)$, imposing that:

$$
N_{U V}^{*}\left(d_{\text {out }}\right) \geq 0.5 \times\left. N_{U V}^{\odot}(1 \mathrm{AU})\right|_{t=t_{A r c}^{\odot}} ^{\odot}
$$

where $N_{U V}^{*}\left(d_{\text {out }}\right)$ is the UV flux at a distance $d_{\text {out }}$ photons emitted by the star in the wavelength $200-315 \mathrm{~nm}$, and $\left.N_{U V}^{\odot}(1 \mathrm{AU})\right|_{t=t_{A r c}^{\odot}}$ is the flux of UV photons received on top of the atmosphere of Primitive Earth when life emerged without any ozone layer protection.

On other the hand, UV radiation could be damaging for biological systems. The destructive effect of the UV radiation on biochemicals processes is usually considered through a biological action spectrum (BAS) $\mathrm{B}(\lambda)$, which represents a relative measure of damage as a function of wavelength. In Buccino et al. (2006), we have defined $\mathrm{B}(\lambda)$ as a function proportional to the probability of a photon of energy $\frac{h c}{\lambda}$ to dissociate free DNA, given by the following expression:

$$
\log B(\lambda) \sim \frac{6.113}{1+\exp \left(\frac{\lambda[n m]-310.9}{8.8}\right)}-4.6 .
$$

Again, applying the Principle of Mediocrity, we set the inner limit $d_{i n}$ of the UV-HZ imposing that:

$$
N_{D N A}^{*}\left(d_{i n}\right) \leq 2 \times\left. N_{D N A}^{\odot}(1 \mathrm{AU})\right|_{t=t_{A r c}^{\odot}} ^{\odot}
$$

where $N_{D N A}^{*}\left(d_{i n}\right)$ is the flux of damaging photons at a distance $d_{i n}$, weighted with $B(\lambda)$, and $\left.N_{D N A}^{\odot}(1 \mathrm{AU})\right|_{t=t_{A r c}^{\odot}}$ is the flux of DNA damaging photons received on Earth 3.8 Gyrs ago. All the atmospheric UV attenuation can be neglected compared to the factor of 2 used due to the Principle of Mediocrity (Buccino et al., 2006).

In Fig. 2 we plot the UV-HZ for the stars under study. We also show the LW-HZ. We note that the LW-HZ around M stars can be wider than the one considered by Kasting et al. (1993). For example, Joshi (2003) reported that the planetary albedo of 
an ocean-covered synchronously- rotating Earth would be $20 \%$ higher than if the planet is not tidally locked. This higher albedo could make the inner limit of the LW-HZ a $10 \%$ closer to the star than the traditional value. On the other hand, the UV radiation of $\mathrm{M}$ stars could lead to a different photochemistry in the planetary atmosphere (richer in $\mathrm{CH}_{4}$ and $\mathrm{N}_{2} \mathrm{O}$ than Earth) and could move away the outer edge of the LW-HZ (Segura et al., 2005). However, this phenomena is not quantified. Therefore, in our LW-HZ calculations we adopt the less restrictive boundaries criteria considered by Kasting et al. (1993), which should be a good approximation for dM stars (for our solar system are 0.75 and $1.77 \mathrm{AU})$.

\section{[Figure 2]}

Laughlin and Bodenheimer (1993) presented several evolutionary tracks which show that the luminosity of stars with masses $0.25-0.35 M_{\odot}$ is almost constant during the main sequence stage. For this reason, we do not need to simulate the temporal evolution of the habitable zones. Both the LW-HZ and the UV-HZ plotted in Fig. 2 would remain constant for near 100 Gyr (Kasting et al. 1993, Tarter et al. 2007). Lowamplitude short-scale variations, no longer than hours, may arise from flare activity, whereas starspots may induce variations of several percent on time-scales from days (rotational modulation of spots) to years (starspots cycle).

For the giant planet Gl876c, the presence of liquid water on a hypothetical moon is possible. Similarly, the exoplanet G1581c, which is a super-Earth with $\sim 5 M_{\oplus}$, resides in the LW-HZ of the star. As pointed in $\$ 1$, this is an important case as it is the planet which most resembles our own Earth. However, neither of these two planets receive enough UV radiation to start the biogenic processes, an alternative energy source would be needed to trigger the formation of complex molecules for the origin of life. This conclusion also applies to any hypothetical terrestrial exoplanet in the LW-HZ around HIP 109388.

On the other hand, much larger UV fluxes can reach the planetary atmosphere during stellar flares, which for dMe stars can be very intense. To study the biological influence of stellar flares, we included in Fig. 2 the UV-HZ and the LW-HZ of two well studied flare stars (AD Leo, M3.5Ve, and EV Lac, M4.5Ve). In both cases, we have estimated the UV-HZ in the quiescent state and for flares of different strength. During the strong flare in AD Leo the UV-HZ increased its width by a factor $\sim 6$, while the inner boundary moved to a position 5.5 times farther than in the quiescent state. In the case of the weaker flare in EV Lac, those factor were 2 and 2.2 respectively. The biological implications of this fact will be discussed in the next Section. 


\section{Discussion}

In the three exoplanetary cases around M stars observed by IUE, the LW-HZ and the UV-HZ are completely separated. Since these stars have low levels of activity, the $\mathrm{UV}-\mathrm{HZ}$ would not present significant variations in time. The UV radiation of these dM stars within the LW-HZ is orders of magnitude smaller than that required to trigger the formation of complex molecules.

In recent years, a strong debate took place in the astrobiological literature about the possibility of panspermia or migration of life-seeds among nearby planets. The distances among dM exoplanets could be shorter than planets around other stellar types, a fact which could increase the chances of panspermia. In this way, life could originate in a planet with enough UV radiation and eventually migrate to a planet with less UV radiation but more moderate temperatures. This could be the case of G1581 and Gl876 planetary systems.

Nevertheless, for the origin of life on an exoplanet in the LW-HZ, a different energy source or an alternative physical mechanism is needed. In principle, flares could provide the energy for the biogenesis. There are large variations regarding the duration, frequency and energy released during stellar flares. In all cases, however, the most energetic impulsive phase of the flare lasts from fractions of a second to a few minutes, and the decay phase, much less energetic, lasts from seconds to many hours. Regarding the flare frequency, it is well known that large flares occur less often and usually last longer than smaller ones (see e.g. Gershberg 2005).

As an example of a moderate flare, we consider the one that took place on EV Lac on September 10 1993 (Pomerance et al. 1 1995). It released a UV flux (in the center of the LW-HZ) of $\mathrm{F}_{U V}(200-315 \mathrm{~nm})=4.94 \times 10^{3} \mathrm{erg} \mathrm{cm}^{2} \mathrm{~s}^{-1}$. For a strong flare, we considered the one observed in AD Leo on April $12^{\text {th }} 1985$, which lasted more than 4 hours, with an abrupt brightness increase and a long decay (Hawley and Pettersen 1991, Mauas and Falchi 1996). At the peak of this flare, a UV flux $\mathrm{F}_{U V}(200-315$ $\mathrm{nm})=4.51 \times 10^{4} \mathrm{erg} \mathrm{cm}^{2} \mathrm{~s}^{-1}$ reached the center of the LW-HZ.

In Fig. 2 we show the impact of these flares on the UV-HZ. It can be seen that during the moderate flare in EV Lac, the UV-HZ coincides with the LW-HZ. Therefore, a moderate flare could provide the UV energy necessary to trigger the biogenic processes. However, since flares are sporadic and of short duration, this energy would be available for only a small fraction of the time. Therefore, it should be much less probable to originate life in a terrestrial planet in the LW-HZ of dM stars.

\section{[Figure 3]}

On the other hand, the UV radiation emitted by the strong flare in AD Leo is several orders of magnitude larger than the radiation needed to destroy biomolecules 
and, therefore, could seriously affect the development of living systems.

However, flares only last a few hours, and most part of this time is spent on its long decay, when the energy emitted is much lower. To illustrate this point, in Fig. 3 we show the UV light curve (at $1 \mathrm{AU}$ ) for this particular flare on AD Leo. It can be seen that the UV flux is already reduced by a factor of 10 about 1 hour after peak time. Also shown in Fig. 3 is the flux of DNA-damaging UV photons $N_{D N A}^{*}$, which evolves with a similar pattern. Moreover, most planets within the LW-HZ around dM stars are probably tidally locked and, therefore, only one face of the planet would receive the damaging UV radiation. Strong flares could provide a strong selective pressure for the emergence and evolution of living organisms, but not necessarily preclude their existence.

On the other hand, it should be kept in mind that strong flares are much less frequent than moderates ones. In particular, the strong AD Leo flare studied here was one of the strongest flares ever recorded (Hawley and Pettersen, 1991). Therefore, mechanisms of DNA repair could operate in the time between damaging flares. In consequence, the intermittent variation of UV radiation emitted by flares could be a source of higher mutation rates. This, in turn, would imply higher microbial biodiversity, and faster adaptation to the changing UV environmental conditions.

Segura et al. (2005) made several simulations of the evolution of atmospheres of hypothetical planets around chromospherically active $M$ stars, and found that the relatively large UV flux could be greatly attenuated by ozone layers similar to the terrestrial one and even thicker (see Table 1 in Scalo et al. 2007). However, their work refers to atmospheric concentrations similar to the present terrestrial one $\left(1 \mathrm{PAL}\right.$ of $\mathrm{O}_{2}$ and $\mathrm{N}_{2}$ ), while life is supposed to have origined with much lower oxygen concentrations.

Another factor to consider is the evolution of chromospheric activity of M-type stars. The usually accepted model to describe the generation and intensification of magnetic fields in late F- to early $\mathrm{M}$-type stars is the $\alpha \Omega$-dynamo first invoked to explain solar activity (Parker 1955). This model, where the large-scale magnetic field generation results from the interaction of differential rotation in the tachocline and the convective turbulence, predicts a strong correlation between activity and rotation. Magnetic activity, which is closely related to stellar rotation, decays with time as the star spins down due to braking by magnetized winds. However, recent results show that this decay happens in the first two Gyr of the stellar life, to be compared with lifetimes for these stars which are at least 25 times larger (Pace and Pasquini, 2004).

This model can only explain the magnetic behaviour of $\mathrm{F}$ to early $\mathrm{M}$ stars, since stars later than M3 are believed to be fully convective and, therefore, cannot sustain a solar like $\alpha \Omega$ dynamo. In particular, the three $\mathrm{M}$ stars with exoplanets (HIP 74995, HIP 109388 and HIP 113020) are at or beyond this limit. Nevertheless, there is plenty of observational evidence that slow late-type rotators like dMe stars are very active and have strong magnetic fields, with filling factors larger than 
for earlier stars (Mochnacki and Zirin 1980, Hawley 1989, Mauas and Falchi 1996). Chabrier and Küker (2006) proposed that for fully convective M stars, large-scale magnetic fields could be produced by a pure $\alpha^{2}$ dynamo, where activity would not decay with time since it does not involve rotation. Although, this model does not predict a cyclic activity, recently Cincunegui et al. (2007) found an activity cycle for Proxima Centauri (M5Ve) with a period of $\sim 1.2$ year. In any case, Prox Cen is a slow rotator, 4-4.5 Gyr old (Demarque et al., 1986), and very active: it presents a flare every 10 hours (Cincunegui et al., 2007).

In summary, our results show that terrestrial-type planets within the LW-HZ around inactive $\mathrm{M}$ stars do not receive enough UV radiation to perform the synthesis of complex macromolecules, and would therefore need an alternative energy source to start the biogenesis. In particular, this is the case of Gl581c, which, according to Udry et al. (2007), is the planet most similar to Earth yet discovered and considered, to date, one of the best candidates for hosting life. In contrast to what it has been believed for a long time, moderate flare activity could play an important role in the origin and evolution of life, triggering the biogenic processes, while the effect of strong flares, that are less frequent, could be mitigated by the fact that most exoplanets within the LW-HZ are probably tidally locked, and therefore only one face of the planet would receive the damaging UV radiation. Taking everything into account, therefore, M stars with moderate flares are the best candidates to host habitable planets.

\section{Acknowledgements}

This research was supported by U401 and X271 UBACYT Research Projects from the University of Buenos Aires and Project 03-12187 of the Agencia de Promoción Científica y Tecnológica (Argentina). G.A.L. was partially supported by The Planetary Society SETI Grant. A.B. was sponsored by a CONICET graduate scholarship.

We thank Sergio Messina and the anonymous Reviewer II for their useful comments and observations.We also thank Antigona Segura and Jill Tarter for their useful comments to an early version of this work.

\section{References}

Benedict, G. F., B. McArthur, E. Nelan, D. Story, A. L. Whipple, P. J. Shelus, W. H. Jefferys, P. D. Hemenway, O. G. Franz, L. H. Wasserman, R. L. Duncombe, W. van Altena, and L. W. Fredrick 1998. Photometry of Proxima Centauri and Barnard's Star Using Hubble Space Telescope Fine Guidance Sensor 3: A Search for Periodic Variations. AJ 116, 429-439.

Bonfils, X., T. Forveille, X. Delfosse, S. Udry, M. Mayor, C. Perrier, F. Bouchy, F. Pepe, D. Queloz, and J.-L. Bertaux 2005. The HARPS search for southern extra-solar planets. VI. A Neptune-mass planet around the nearby M dwarf Gl 581. 
$A \mathscr{G}$ A 443, L15-L18.

Buccino, A. P., G. A. Lemarchand, and P. J. D. Mauas 2006. Ultraviolet radiation constraints around the circumstellar habitable zones. Icarus 183, 491-503.

Butler, R. P., J. A. Johnson, G. W. Marcy, J. T. Wright, S. S. Vogt, and D. A. Fischer 2006. A Long-Period Jupiter-Mass Planet Orbiting the Nearby M Dwarf GJ 849. PASP 118, 1685-1689.

Cassatella, A., A. Altamore, R. González-Riestra, J. D. Ponz, J. Barbero, A. Talavera, and W. Wamsteker 2000. The INES system. II. Ripple correction and absolute calibration for IUE high resolution spectra. Astron. Astrophys. Suppl. Ser. 141, 331-341.

Chabrier, G., and M. Küker 2006. Large-scale $\alpha^{2}$-dynamo in low-mass stars and brown dwarfs. A\&A 446, 1027-1037.

Cincunegui, C. C., R. Diaz, and P. Mauas 2007. A possible activity cycle in Prox Cen. A\&SA 461, 1107-1113.

Cockell, C. S. 1998. Biological effect of High Ultraviolet Radiation on early Earth- a Theorical Evaluation. J. theor.Biol. 193, 717-729.

Deeg, H. J. 2004. Should Eddington concentrate on M stars? In F. Favata, S. Aigrain, and A. Wilson (Eds.), ESA SP-538: Stellar Structure and Habitable Planet Finding, pp. 231-237.

Demarque, P., D. B. Guenther, and W. F. van Altena 1986. The case of Alpha Centauri - Mass, age and p-mode oscillation spectrum. ApJ 300, 773-778.

Dole, S. H. 1964. Habitable planets for man. New York, Blaisdell Pub. Co. 1964 [1st ed.].

Ehrenfreund, P., W. Irvine, L. Becker, J. Blank, J. R. Brucato, L. Colangeli, S. Derenne, D. Despois, A. Dutrey, H. Fraaije, A. Lazcano, T. Owen, and F. Robert 2002. Astrophysical and astrochemical insights into the origin of life. Reports of Progress in Physics 65, 1427-1487.

Endl, M., W. D. Cochran, R. G. Tull, and P. J. MacQueen 2003. A Dedicated M Dwarf Planet Search Using The Hobby-Eberly Telescope. AJ 126, 3099-3107.

Gershberg, R. E. 2005. Solar-Type Activity in Main-Sequence Stars. Springer, Heidelberg, The Netherlands.

Haberle, R. M., C. P. McKay, D. Tyler, and R. T. Reynolds 1996. Can Synchronously Rotating Planets Support An Atmosphere? In L. R. Doyle (Ed.), Circumstellar Habitable Zones, pp. 29. 
Hawley, S. L. 1989. As observational and theoretical investigation of stellar flares. Ph. D. thesis, AA(Texas Univ., Austin.).

Hawley, S. L., and B. R. Pettersen 1991. The great flare of 1985 April 12 on AD Leonis. ApJ 378, 725-741.

Heath, M. J., L. R. Doyle, M. M. Joshi, and R. M. Haberle 1999. Habitability of Planets Around Red Dwarf Stars. Origins of Life and Evolution of the Biosphere 29, $405-424$.

Johns-Krull, C. M., and J. A. Valenti 1996. Detection of strong magnetic fields on M dwarfs. ApJ 459, L95-L98.

Joshi, M. 2003. Climate Model Studies of Synchronously Rotating Planets. Astrobiology $3,415-427$.

Joshi, M. M., R. M. Haberle, and R. T. Reynolds 1997. Simulations of the Atmospheres of Synchronously Rotating Terrestrial Planets Orbiting M Dwarfs: Conditions for Atmospheric Collapse and the Implications for Habitability. Icarus 129, 450-465.

Kasting, J. F., D. P. Whitmire, and R. T. Reynolds 1993. Habitable Zones around Main Sequence Stars. Icarus 101, 108-128.

Khodachenko, M. L., I. Ribas, H. Lammer, J.-M. Grießmeier, M. Leitner, F. Selsis, C. Eiroa, A. Hanslmeier, H. K. Biernat, C. J. Farrugia, and H. O. Rucker 2007. Coronal Mass Ejection (CME) Activity of Low Mass M Stars as An Important Factor for The Habitability of Terrestrial Exoplanets. I. CME Impact on Expected Magnetospheres of Earth-Like Exoplanets in Close-In Habitable Zones. Astrobiology $7,167-184$.

Kiraga, M., and K. Stepien 2007. Age-Rotation-Activity Relations for M Dwarf Stars Based on ASAS Photometric Data. ArXiv e-prints 707.

Laughlin, G., and P. Bodenheimer 1993. Luminosity functions for very low mass stars and brown dwarfs. ApJ 403, 303-314.

Laughlin, G., P. Bodenheimer, and F. C. Adams 1997. The End of the Main Sequence. ApJ 482, 420-+.

Lindberg, C., and G. Horneck 1991. Action spectra for survival and spore photoproduct formation of Bacillus subtilis irradiated with short-wavelength (200-300 nm) UV at atmospheric pressure and in vacuo. J. Photochem. Photobiol. B: Biol. 11, 69-80.

Massa, D., and E. L. Fitzpatrick 1998. A recalibration of IUE NEWSIPS low dispersion data. Bulletin of the American Astronomical Society 30, 1122-1267. 
Mauas, P. J. D., and A. Falchi 1996. Atmospheric models of flare stars: the flaring state of AD Leonis. A\&A 310, 245-258.

Mochnacki, S. W., and H. Zirin 1980. Multichannel spectrophotometry of stellar flares. ApJL 239, L27-L31.

Pace, G., and L. Pasquini 2004. The age-activity-rotation relationship in solar-type stars. A\&A 426, 1021-1034.

Parker, E. N. 1955. Hydromagnetic Dynamo Models. ApJ 122, 293-314.

Pomerance, B. H., B. Abbott, and C. Ambruster 1995. Simultaneous EUVE and IUE Flares from the Flare Star EV Lac. In Bulletin of the American Astronomical Society, Volume 27, pp. 838.

Rodonó, M. 1986. The atmospheres of $M$ dwarfs: Observations, pp. 409-453. NASA, Washington, The M-Type Stars.

Scalo, J., L. Kaltenegger, A. G. Segura, M. Fridlund, I. Ribas, Y. N. Kulikov, J. L. Grenfell, H. Rauer, P. Odert, M. Leitzinger, F. Selsis, M. L. Khodachenko, C. Eiroa, J. Kasting, and H. Lammer 2007. M Stars as Targets for Terrestrial Exoplanet Searches And Biosignature Detection. Astrobiology 7, 85-166.

Schneider, J. 2007. The Extrasolar Planets Encyclopaedia. http://exoplanet.eu.

Segura, A., J. F. Kasting, V. Meadows, M. Cohen, J. Scalo, D. Crisp, R. A. H. Butler, and G. Tinetti 2005. Biosignatures from Earth-Like Planets Around M Dwarfs. Astrobiology 5, 706-725.

Tarter, J. C., P. R. Backus, R. L. Mancinelli, J. M. Aurnou, D. E. Backman, G. S. Basri, A. P. Boss, A. Clarke, D. Deming, L. R. Doyle, E. D. Feigelson, F. Freund, D. H. Grinspoon, R. M. Haberle, S. A. Hauck, II, M. J. Heath, T. J. Henry, J. L. Hollingsworth, M. M. Joshi, S. Kilston, M. C. Liu, E. Meikle, I. N. Reid, L. J. Rothschild, J. Scalo, A. Segura, C. M. Tang, J. M. Tiedje, M. C. Turnbull, L. M. Walkowicz, A. L. Weber, and R. E. Young 2007. A Reappraisal of The Habitability of Planets around M Dwarf Stars. Astrobiology 7, 30-65.

Toupance, G., A. Bossard, and F. Raulin 1977. Far UV irradiation of model prebiotic atmospheres. Origins of Life and Evolution of Biospheres 8, 259-266.

Udry, S., X. Bonfils, X. Delfosse, T. Forveille, M. Mayor, C. Perrier, F. Bouchy, C. Lovis, F. Pepe, D. Queloz, and J. . Bertaux 2007. The HARPS search for southern extra-solar planets XI. Super-Earths (5\& 8 M_Earth) in a 3-planet system. A\& A 469, L43-L47. 
von Bloh, W., C. Bounama, M. Cuntz, and S. Franck 2007. The habitability of super-Earths in Gliese 581. ArXiv e-prints 705.

von Hoerner, S. 1961. The search for signals from other civilizations. Science 134, $1839-1843$.

Wetherill, G. W. 1996. The Formation and Habitability of Extra-Solar Planets. Icarus 119, 219-238. 


\section{Tables}

Table 1

Physical Parameters of M stars with exoplantes observed by IUE and their planets.

\begin{tabular}{|c|c|c|c|c|c|c|c|c|c|}
\hline \multicolumn{6}{|c|}{ Stellar Properties } & \multicolumn{4}{|c|}{ Planetary Properties } \\
\hline Star & Sp. Type & Mass & dist. & $\mathrm{m}_{V}$ & Age & Planet & $\operatorname{Mass}[\sin i]$ & Semimajor & Period \\
\hline HIP & and Class & $\left(\mathrm{M}_{\odot}\right)$ & (pc) & & (Gyr.) & & $\left(\mathrm{M}_{J}\right)$ & Axis $(\mathrm{AU})$ & (days) \\
\hline \multirow[t]{3}{*}{74995} & M3V & 0.31 & 6.26 & 10.55 & 4.30 & G1581b & 0.060 & 0.04 & 5.40 \\
\hline & & & & & & Gl581c & 0.016 & 0.07 & 12.93 \\
\hline & & & & & & Gl581d & 0.024 & 0.25 & 83.60 \\
\hline 109388 & $\mathrm{M} 3.5 \mathrm{~V}$ & 0.36 & 8.80 & 10.42 & - & Gj849b & 0.820 & 2.35 & 1890.00 \\
\hline \multirow[t]{3}{*}{113020} & M4V & 0.32 & 4.72 & 10.17 & 9.90 & Gl876b & 1.940 & 0.21 & 60.94 \\
\hline & & & & & & Gl876c & 0.560 & 0.13 & 30.10 \\
\hline & & & & & & Gl876d & 0.020 & 0.02 & 1.94 \\
\hline
\end{tabular}




\section{FIGURES CAPTIONS}

Caption Fig. 19: IUE Spectra of F to M dwarfs stars: HD 9826 (F8V, short dash), 18 Sco (G2V, solid), HD 3651 (K0V, long dash), AD Leo quiescent (M3.5V, dot-long dash), AD Leo with flare (M3.5Ve, dot-short dash), and, with dotted line, the M stars with exoplanets HIP 109388 (M3.5V), HIP 113020 (M4V) and HIP 74995 (M3V). The last two spectra are arbitrary displaced for clarity by $10^{-2}$ and $10^{-4} \mathrm{erg} \mathrm{cm}^{-2} \mathrm{~s}^{-1} \AA^{-1}$ respectively.

Caption Fig. 22 Habitable zones around the planetary stars HIP 109388 (Gl 581), HIP 74995 (Gj 849) and HIP 113020 (Gl 876) and around the flare stars AD Leo and EV Lac in the quiescent and flaring states. The solid lines represent the LW-HZ, the rectangles are the UV-HZ and the triangular dots indicate the positions of the exoplanets.

Caption Fig. 35 The UV flux at $1 \mathrm{AU}(\mathbf{\Lambda})$ and the corresponding flux of DNAdamaging UV photons $N_{D N A}^{*}$ at $1 \mathrm{AU}(\bullet)$ of AD Leo flare of April, $12^{\text {th }} 1985$. 
FIGURES

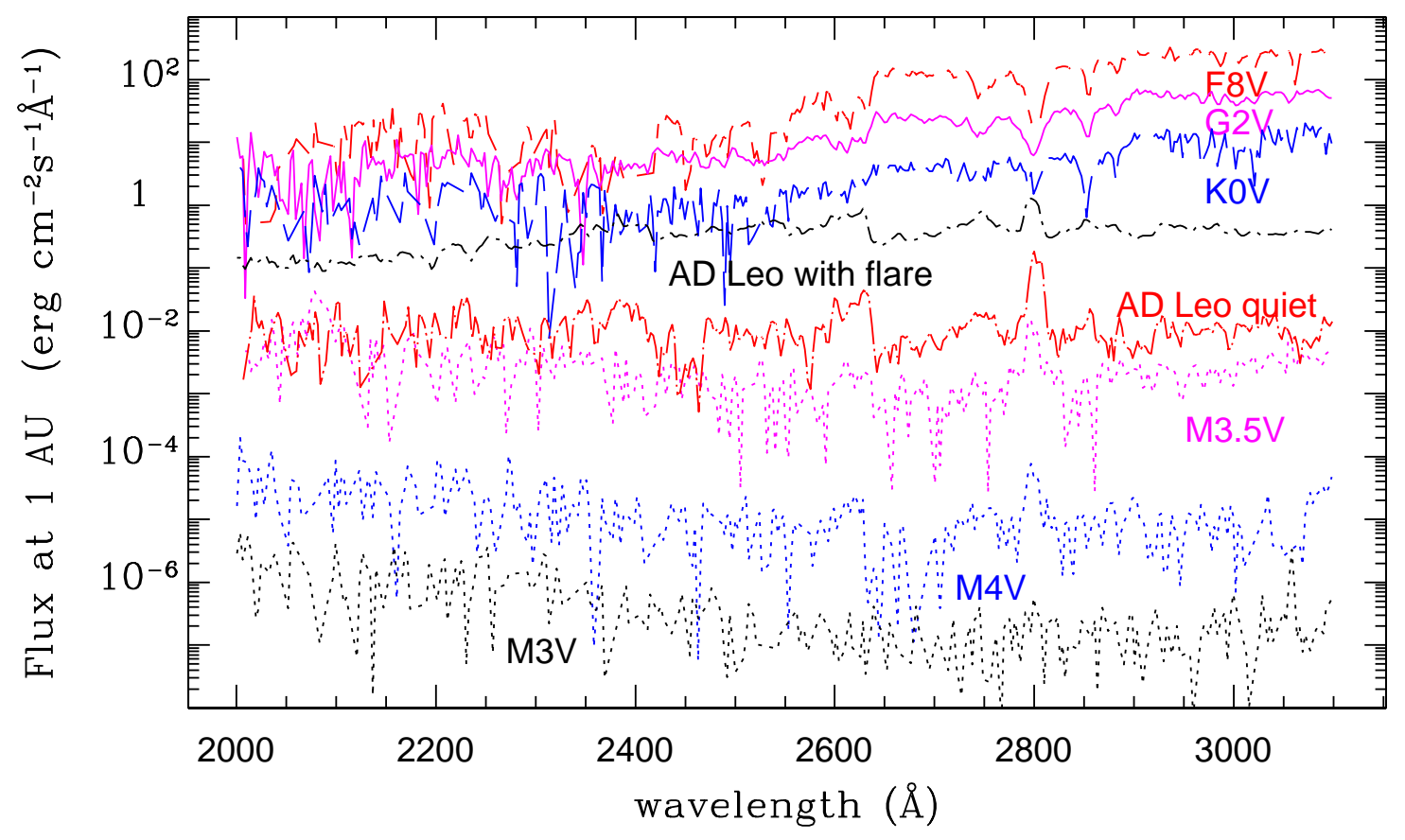

Fig. 1. Buccino et al., 2007. IUE spectra of M stars.

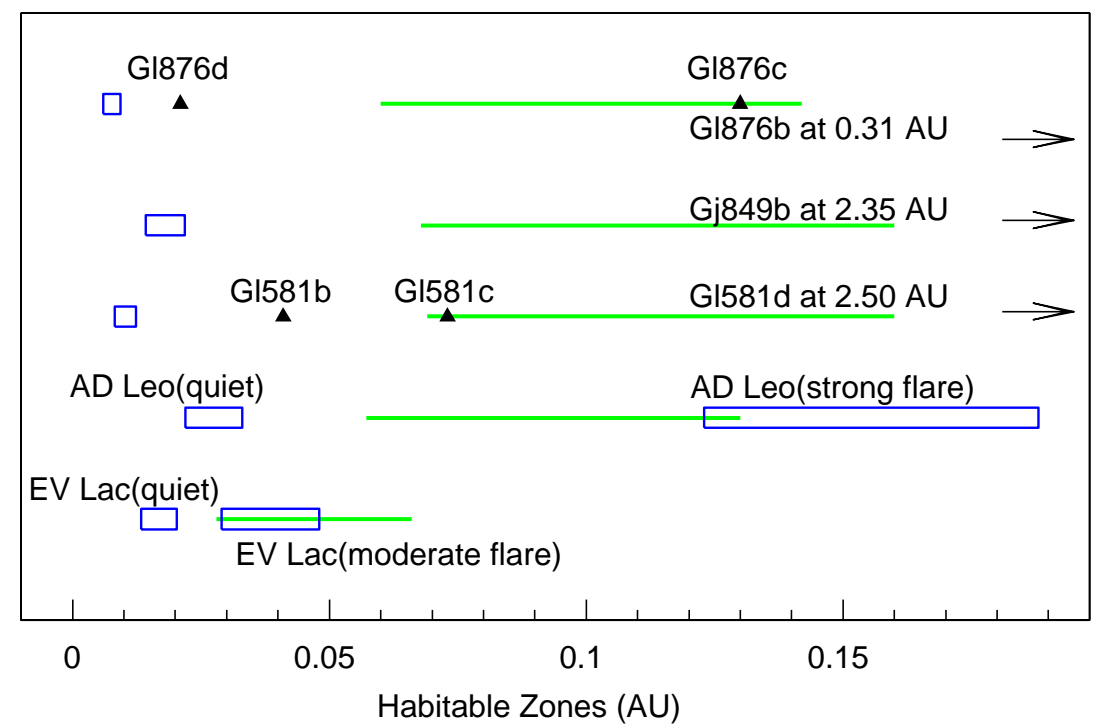

Fig. 2. Buccino et al., 2007. Habitable Zones around M stars. 


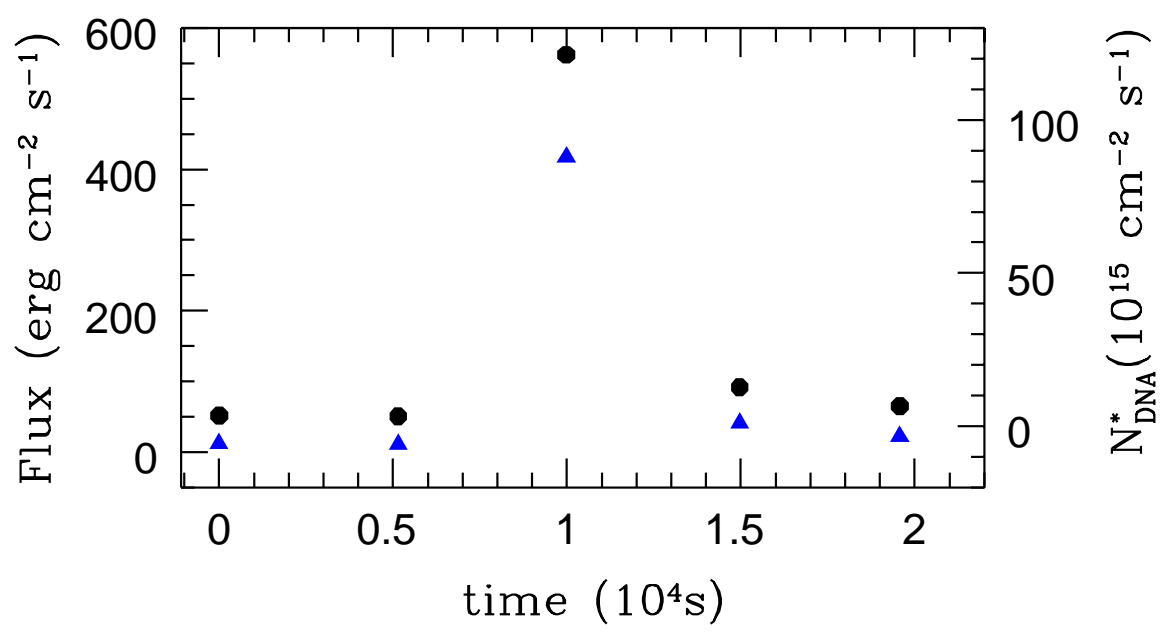

Fig. 3. Buccino et al., 2007. Flare in AD Leo. 